\title{
Proposed New Antiproton Experiments at Fermilab
}

\author{
Daniel M. Kaplan
}

Received: date / Accepted: date

\begin{abstract}
Fermilab operates the world's most intense source of antiprotons. Recently various experiments have been proposed that can use those antiprotons either parasitically during Tevatron Collider running or after the Tevatron Collider finishes in about 2010. We discuss the physics goals and prospects of the proposed experiments.
\end{abstract}

Keywords Antiproton - Antihydrogen - Antimatter · Charm · Charmonium - CPT · Gravity · Hyperons

\section{Introduction}

The world's highest-energy and highest-intensity antiproton source is at Fermilab (see Table 1). Having previously supported medium-energy antiproton fixed-target experiments (including the charmonium experiments E760 and E835), it is now 100\% dedicated to providing luminosity for the Tevatron Collider. At CERN, the LEAR antiproton storage ring was decommissioned in $1996{ }^{1}$ its successor facility, the Antiproton Decelerator $(\mathrm{AD})$, provides antiproton beams at momenta of 100 and $300 \mathrm{MeV} / c$, at intensities up to $\approx 2 \times 10^{7}$ per minute 1 . It is noteworthy that Germany has embarked on a $\approx$ billion-Euro upgrade plan for the GSI-Darmstadt nuclear-physics laboratory that includes construction of 30 and $90 \mathrm{GeV}$ rapid-cycling synchrotrons and low- and medium-energy antiproton storage rings 2 . These facilities address an interesting and varied research program in nuclear and particle physics and beyond.

To appear in Proceedings of IXth International Conference on Low Energy Antiproton Physics (LEAP'08), Vienna, Austria, September 16 to 19, 2008.

\section{M. Kaplan}

Illinois Institute of Technology

Tel.: +1 312-567-3389

Fax: +1 312-567-3289

E-mail: kaplan@iit.edu

1 LEAR was turned off in spite of its review committee's recommendation that it be allowed to complete its planned program of research; the rationale was to free up expert manpower for LHC work. The "ground rules" for the AD design accordingly required operability by as small a crew as possible. 
Table 1 Antiproton energies and intensities at existing and future facilities.

\begin{tabular}{lccccc}
\hline \hline \multirow{2}{*}{ Facility } & $\bar{p}$ K.E. & \multicolumn{2}{c}{ Stacking: } & Hours & $\bar{p} / \mathrm{Yr}$ \\
& $(\mathrm{GeV})$ & Rate $\left(10^{10} / \mathrm{hr}\right)$ & Duty Factor & $/$ Yr & $\left(10^{13}\right)$ \\
\hline \hline CERN AD & $0.005,0.047$ & - & - & 3800 & 0.4 \\
FNAL (Accumulator) & $\approx 3.5-8$ & 20 & $15 \%$ & 5550 & 17 \\
FNAL (New Ring) & $2-20 ?$ & 20 & $90 \%$ & 5550 & 100 \\
FAIR ( $(2015)$ & $2-15$ & 3.5 & $90 \%$ & $2780^{*}$ & 9 \\
\hline \hline
\end{tabular}

* The lower number of operating hours at FAIR compared with that at other facilities arises from medium-energy antiproton operation having to share time with other programs.

\section{Physics Overview}

A number of intriguing recent discoveries can be elucidated at such a facility, foremost among which is charm mixing [3]. The key question is whether there is new physics in charm mixing; the signature for this is $C P$ violation [4. The search for new physics in $B$ and $K$ mixing and decay has so far come up empty. Thus it behooves us to look elsewhere as well. As pointed out by many authors, charm is an excellent venue for such investigation: It is the only up-type quark for which such effects are possible, and standard-model backgrounds to new physics in charm are suppressed by small CKMmatrix elements and the fact that the $b$ quark is the most massive one participating in loop diagrams [5]. We argue below that a charm experiment at the Fermilab Antiproton Source might be the world's most sensitive.

Other topics of interest include such states as the $X(3872)$ in the charmonium region [6], observed by several groups, as well as the investigation of possible newphysics signals observed in the HyperCP experiment at Fermilab: evidence for $C P$ violation [7] and flavor-changing neutral currents 8] in hyperon decay. In addition, the $h_{c}$ mass and width, $\chi_{c}$ radiative-decay angular distributions, and $\eta_{c}^{\prime}(2 S)$ full and radiative widths, important parameters of the charmonium system that remain to be precisely determined [9, are well suited to the $\bar{p} p$ technique [10].

Charm particles can be pair-produced in $\bar{p} p$ or $\bar{p} N$ collisions at and above the $\psi(3770)$ resonance. There is an enormous cross-section advantage relative to $e^{+} e^{-}$ colliders: charm hadroproduction cross sections are typically $\mathcal{O}(10 \mu \mathrm{b})$, while $e^{+} e^{-}$ cross sections are $\mathcal{O}(1 \mathrm{nb})$. Against this must be weighed the $e^{+} e^{-}$luminosity advantage, typically $\mathcal{O}\left(10^{2}\right)$, and the lower background rates in $e^{+} e^{-}$experiments. Charm hadroproduction at high energies comes with the advantage of longer decay distances, but the countervailing disadvantage of higher multiplicity $\left(\left\langle n_{c h}\right\rangle \sim 10\right)$ in the underlying event. We expect that the low charged-particle multiplicity $\left(\left\langle n_{c h}\right\rangle \approx 2\right)$ in $\bar{p} p$ collisions somewhat above open-charm threshold will enable charm samples with cleanliness comparable to that at the $B$ factories, with the application of only modest cuts, and hence, high efficiency. The competition for this program is a possible "super- $B$ factory."

By scanning the Antiproton Accumulator beam energy across the resonance, Fermilab experiments E760 and E835 made the world's most precise measurements of charmonium masses and widths [10. Besides this precision, the other key advantage of the antiproton-annihilation technique is its ability to produce charmonium states of all quantum numbers, in contrast to $e^{+} e^{-}$machines which produce primarily $1^{--}$states and the few states that couple directly to them, or (with relatively low statistics) states accessible in $B$ decay or in $2 \gamma$ production. 


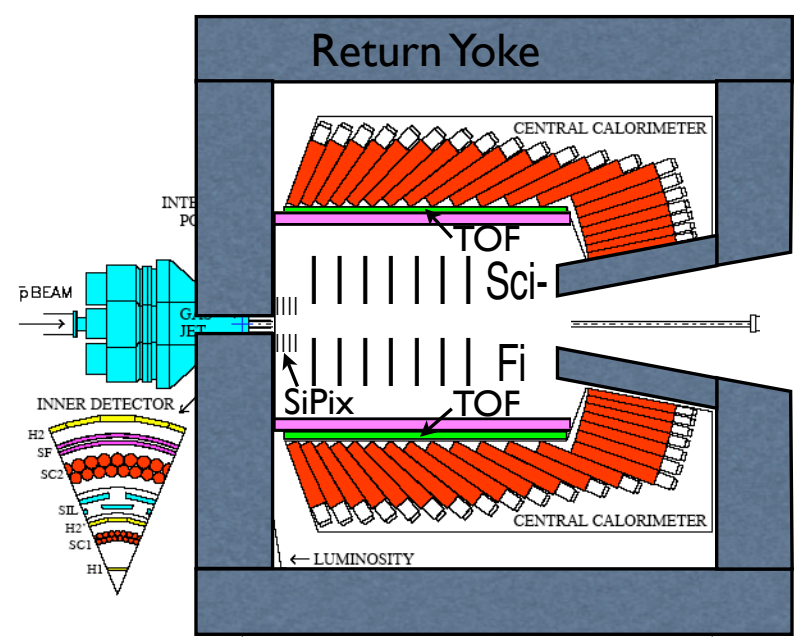

Fig. 1 Sketch of upgraded E835 apparatus as discussed in text: 1 T solenoid shown in magenta, TOF counters in green. Return yoke (needed to assure operability of calorimeter phototubes) should consist of as little iron as necessary.

The E835 apparatus did not include a magnet, thus various cross sections needed to assess the performance and reach of a new experiment remain unmeasured. However, they can be estimated with some degree of confidence. We are proposing to assemble, quickly and at modest cost, an "upgraded E835" spectrometer that includes a magnet. If these cross sections are of the expected magnitude, it should be possible with this apparatus to make the world's best measurements of charm mixing and $C P$ violation, as well as of the other effects mentioned above. (To take full advantage of the capabilities of the Fermilab Antiproton Source, a follow-on experiment in a new, dedicated ring à la Table 1 might then be designed for even greater sensitivity.)

\section{Proposed antiproton experiments at Fermilab}

\subsection{Medium-energy $\bar{p} p$-annihilation experiment}

By adding a small magnet, tracking and vertex detectors, and TOF counters to the E835 calorimeter as in Fig. 1 plus modern, high-bandwidth triggering and dataacquisition systems, several important topics can be studied. We assume $\bar{p} p$ or $\bar{p} N$ luminosity of $2 \times 10^{32} \mathrm{~cm}^{-2} \mathrm{~s}^{-1}$, one order of magnitude beyond that of E835, which can be accomplished by use of a denser internal target than the E835 hydrogen cluster jet 10 .

Charm mixing and CP violation After a more than 20-year search, $D^{0}-\bar{D}^{0}$ mixing is now established at 9.2 standard deviations [3], thanks mainly to the $B$ factories. The level of mixing is consistent with the wide range of standard-model predictions 4; however, this does not preclude a significant and potentially detectable contribution from new physics 11. Since some new-physics models predict different effects in the charge-2/3 ("up-type") quark sector than in the down-type [11, it is important to 
carry out such studies of charm mesons - the only up-type system for which meson mixing can occur.

The $\bar{p} p$ annihilation cross section to open charm could be substantial; for example, a recent estimate gives $\sigma\left(\bar{p} p \rightarrow D^{* 0} \bar{D}^{0}\right) \approx 1.3 \mu \mathrm{b}$ at $\sqrt{s}=4.2 \mathrm{GeV} 12$. At $\mathcal{L}=$ $2 \times 10^{32} \mathrm{~cm}^{-2} \mathrm{~s}^{-1}$, this represents some $5 \times 10^{9}$ events per year, substantially exceeding each year the integrated sample $\left(10^{9}\right.$ events) available at the $B$ factories. Since there will also be $D^{* \pm} D^{\mp}, D^{*} \bar{D}^{*}$, and $D \bar{D}$ events, the total charm sample will be even larger; with the use of a target nucleus heavier than hydrogen, the charm-production $A$-dependence 13. could enhance statistics by an additional factor of a few. Such a target could also localize primary interactions to an $\mathcal{O}(\mu \mathrm{m})$-sized region, allowing the $D$-meson decay distance to be cleanly resolved. Medium-energy $\left(p_{\bar{p}} \approx 8 \mathrm{GeV} / c\right) \bar{p} N$ annihilation may thus be the optimal way to study charm mixing and search for possible new-physics contributions via the clean signature [14] of charm $C P$ violation (CPV).

Hyperon CP violation and rare decays The Fermilab HyperCP Experiment [15] amassed the world's largest samples of hyperon decays, including $2.5 \times 10^{9}$ reconstructed $\left(\Xi^{\prime} \mp\right.$ decays and $10^{10}$ produced $\Sigma^{+}$. HyperCP observed unexpected signals at the $\gtrsim 2 \sigma$ level for possible new physics in the rare hyperon decay $\Sigma^{+} \rightarrow p \mu^{+} \mu^{-}$. ${ }^{(} \bar{\Xi}^{)} \mp \rightarrow{ }^{(} \bar{\Lambda}^{\prime} \pi^{\mp} \rightarrow{ }^{(} \bar{p}^{\prime} \pi^{\mp} \pi^{\mp} C P$ asymmetry 7 . Since the $\bar{p} p \rightarrow \Omega^{-} \bar{\Omega}^{+}$threshold lies in the same region as the open-charm threshold, the proposed experiment can further test these observations using ${ }^{(} \bar{\Omega}{ }^{\prime} \mp \rightarrow{ }^{(} \Xi^{\prime} \mp \mu^{+} \mu^{-}$decay and potential ${ }^{(} \bar{\Omega}{ }^{\prime} \mp \mathrm{CPV}[16$. While the $\bar{p} p \rightarrow \Omega^{-} \bar{\Omega}^{+}$cross section has not been measured, by extrapolation from $\bar{p} p \rightarrow \Lambda \bar{\Lambda}$ and $\bar{p} p \rightarrow \Xi^{-\bar{\Xi}^{+}}$one obtains an estimate just above threshold of $\approx 60 \mathrm{nb}$, implying $\sim 10^{8}$ events produced per year. In addition the measured $\approx 1 \mathrm{mb}$ cross section for associated production of inclusive hyperons [17] would mean $\sim 10^{12}$ events produced per year, which could directly confront the HyperCP evidence (at $\approx 2.4 \sigma$ significance) for a possible new particle of mass $214.3 \mathrm{MeV} / c^{2}$ in the three observed $\Sigma^{+} \rightarrow p \mu^{+} \mu^{-}$events (Fig. $22^{2}$ Further in the future, the dedicated $\bar{p}$ storage ring of Table 1 could decelerate antiprotons to the $\Lambda \bar{\Lambda}, \Sigma^{+} \bar{\Sigma}^{-}$, and $\Xi^{-} \bar{\Xi}^{+}$thresholds, where an experiment at $10^{33}$ luminosity could amass the clean, $>10^{10}$-event samples needed to confirm or refute the HyperCP evidence for $C P$ asymmetry in $\Xi^{ \pm}$decay [7].

Precision charmonium measurements Using the Fermilab Antiproton Source, experiments E760 and E835 made the world's most precise measurements of charmonium masses and widths 10. Although charmonium has by now been extensively studied, a number of questions remain, including the nature of the mysterious $X(3872)$ state [6] and improved measurement of $h_{c}$ and $\eta_{c}^{\prime}$ parameters [9. The unique precision of the $\bar{p} p$ energy-scan technique is ideally suited to making the precise mass and width measurements needed to test the intriguing hypothesis that the $X(3872)$ is a $D^{* 0} \bar{D}^{0}$ molecule [19].

\subsection{Antihydrogen experiments}

Antihydrogen-in-flight CPT tests The study of antihydrogen atoms in flight may be a way around some of the difficulties encountered in the CERN trapping experiments.

\footnotetext{
2 Such a particle, if confirmed, could be evidence for nonminimal SUSY 18 .
} 

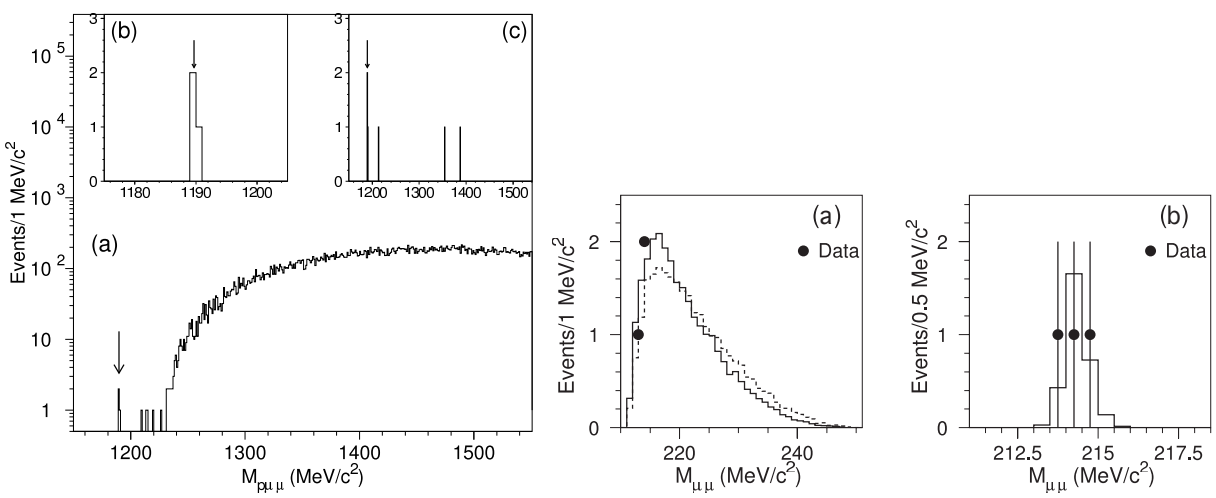

Fig. 2 (Left) Mass spectrum for 3-track final states consistent with being single-vertex $p \mu^{+} \mu^{-}$ events in HyperCP positive-beam data sample: (a) wide mass range (semilog scale); (b) narrow range around $\Sigma^{+}$mass; (c) after application of additional cuts as described in Ref. [8]. (Arrows indicate mass of $\Sigma^{+}$.) (Right) Dimuon mass spectrum of the three HyperCP $\Sigma^{+} \rightarrow p \mu^{+} \mu^{-}$ candidate events compared with Monte Carlo spectrum assuming (a) SM virtual-photon form factor (solid) or isotropic decay (dashed), or (b) decay via a narrow resonance $X^{0}$.

First steps in this direction were taken by PS210 at LEAR [20] and Fermilab E862 [21], which observed formation of antihydrogen in flight in the mid-1990s. Methods to measure the antihydrogen Lamb shift and fine structure (the $2 s_{1 / 2}-2 p_{1 / 2}$ and $2 p_{1 / 2}-2 p_{3 / 2}$ energy differences) were subsequently worked out 22 . Progress toward this goal may be compatible with normal Tevatron Collider operations - a possibility currently under investigation. If the feasibility of the approach is borne out by further work, the program could continue into the post-Tevatron era. Sensitivity at the parts-per-billion level ( $\sim 10^{-9}$ of the $2 S$ energy) may be possible - not the $\sim 10^{-14}$ envisioned for the $\mathrm{AD}$ program [23] but a valuable first step.

Antimatter gravity experiment While General Relativity predicts that the gravitational forces on matter and antimatter should be identical, no direct experimental test of this prediction has yet been made 24 . Attempts at a quantized theory of gravity generally introduce non-tensor forces, which could cancel for matter-matter and antimatter-antimatter interactions but add for matter-antimatter ones. In addition, possible "fifth forces" or non- $1 / r^{2}$ dependence have been discussed. Such effects can be sensitively sought by measuring the gravitational acceleration of antimatter in the field of the earth. While various such experiments have been discussed for many years, one - measurement of the gravitational acceleration of antihydrogen - has only recently become feasible and is now proposed both at CERN and at Fermilab 23 25.

The principle of the Antimatter Gravity Experiment (AGE) is to form a beam of slow $(\approx 1 \mathrm{~km} / \mathrm{s})$ antihydrogen in a Penning trap and pass the beam through a $\approx 1$ m-long Mach-Zehnder interferometer. The phase of the interference pattern can be measured to a small fraction of the $(\approx 1 \mu \mathrm{m})$ grating period, and measurement of the phase vs. the speed of the atom determines $\bar{g}$. Simulation shows that $\bar{g}$ can be measured to $0.6 \%$ of $g$ with one-million antihydrogen $(\bar{H})$ atoms incident on the interferometer; this could be done parasitically during the Tevatron run. The proposed AGE goal is a $10^{-4}$ measurement, requiring $10^{10} \bar{H}$ atoms. Given the expected $\gtrsim 10^{-5}$ antiprotontrapping and $\bar{H}$-formation efficiency, this can be accomplished at the Antiproton Source in a few-month dedicated run. 


\section{Outlook}

With the end of the Tevatron Collider program in sight, new and unique measurements are possible at the Fermilab Antiproton Source [26]. Such a program can substantially broaden the clientele and appeal of US particle physics.

Acknowledgements The author thanks his pbar collaborators ${ }^{3}$ and E. Braaten, E. Eichten, and C. Quigg for useful conversations. Work supported by Department of Energy grant DEFG02-94ER40840.

\section{References}

1. T. Eriksson, in Proc. LEAP05 Conf., AIP Conf. Proc. 796, 389 (2005).

2. See http://www.gsi.de/fair/index_e.html

3. E. Barberio et al., arXiv:0808.1297 [hep-ex].

4. See e.g. I. I. Bigi and N. Uraltsev, Nucl. Phys. B 592 (2001) 92

5. See e.g. Sec. 3.9 of G. Buchalla et al., Report of Working Group 2 of the CERN Workshop "Flavour in the era of the LHC," Geneva, Switzerland, November 2005 - March 2007, arXiv:0801.1833 [hep-ph] (2008).

6. E. Eichten. K, Lane, and C. Quigg, Phys. Rev. D 73, 014014 (2006); Erratum-ibid. D 73, 079903 (2006).

7. C. Materniak, presented at the BEACH08 Conference, Columbia, SC, 22-28 June 2008, http://beach2008.sc.edu/includes/documents/sessions/materniak.talk.pdf

8. H. K. Park et al., Phys. Rev. Lett. 94, 021801 (2005).

9. N. Brambilla et al. [Quarkonium Working Group], Heavy Quarkonium Physics, CERN Yellow Report CERN-2005-005 (2005).

10. G. Garzoglio et al., Nucl. Instr. Meth. A 519, 558 (2004); T. A. Armstrong et al., Phys. Rev. D 47, 772 (1993).

11. See e.g. Y. Grossman, A. L. Kagan, Y. Nir, Phys. Rev. D 75, 036008 (2007).

12. E. Braaten, Phys. Rev. D 77, 034019 (2008).

13. M. J. Leitch et al., Phys. Rev. Lett. 72, 2542 (1994).

14. See e.g. A. A. Petrov, arXiv:0806.2498v1 [hep-ph], and references therein.

15. R. A. Burnstein et al., Nucl. Instr. Meth. A 541, 516 (2005).

16. J. Tandean, Phys. Rev. D 70, 076005 (2004); J. Tandean and G. Valencia, Phys. Lett. B 451, 382 (1999).

17. C. Y. Chien et al., Phys. Rev. 152, 1181 (1066).

18. D. S. Gorbunov, V. A. Rubakov, Phys. Rev. D 64, 054008 (2001); D. S. Gorbunov, Nucl. Phys. B 602, 213 (2001); X.-G. He, J. Tandean, G. Valencia, Phys. Lett. B 631, 100 (2005).

19. N. A. Tørnqvist, Phys. Lett. B 590, 209 (2004).

20. G. Baur et al., Phys. Lett. B 368, 251 (1996).

21. G. Blanford et al., Phys. Rev. Lett. 80, 3037 (1998).

22. G. Blanford et al., Phys. Rev. D 57, 6649 (1998).

23. AEGIS Proposal, G. Drobychev et al., CERN-SPSC-2007-017.

24. M. Fischler, J. Lykken, T. Roberts, FERMILAB-FN-0822-CD-T, arXiv:0808.3929 [hep-th].

25. AGE Letter of Intent, A. D. Cronin et al., http://capp.iit.edu/hep/pbar/AGE_FNAL08_LoI_v1_4.pdf

26. See "New pbar Experiments for Fermilab" web page: http://capp.iit.edu/hep/pbar/

3 Arizona: A. Cronin; Riverside: A. P. Mills, Jr.; Cassino: G. M. Piacentino; Duke: T. J. Phillips; FNAL: G. Apollinari, D. R. Broemmelsiek, B. C. Brown, C. N. Brown, D. C. Christian, P. Derwent, M. Fischler, K. Gollwitzer, A. Hahn, V. Papadimitriou, R. Stefanski, J. Volk, S. Werkema, H. B. White, G. P. Yeh; Ferrara: W. Baldini, G. Stancari, M. Stancari; Hbar Tech.: J. R. Babcock, S. D. Howe, G. P. Jackson, J. M. Zlotnicki; IIT: D. M. Kaplan, T. J. Roberts, H. A. Rubin, Y. Torun, C. G. White; KSU: G. A. Horton-Smith, B. Ratra; KyungPook: H. K. Park; Luther Coll.: T. K. Pedlar; Michigan: H. R. Gustafson, M. Longo, D. Rajaram; Northwestern: J. Rosen; Notre Dame: M. Wayne; SMU: T. Coan; SXU: A. Chakravorty; Virginia: E. C. Dukes; Wayne State: G. Bonvicini. 